EPJ manuscript No.

(will be inserted by the editor)

\title{
From chaos of lines to Lagrangian structures in flux conservative fields
}

\author{
Xavier Leoncini ${ }^{1,2}$, Olivier Agullo ${ }^{2}$, Magali Muraglia ${ }^{2}$, and Cristel Chandre ${ }^{1}$ \\ 1 Centre de Physique Théorique ${ }^{a}$, Université de Provence-CNRS Luminy, Case 907 F-13288 Marseilles cedex 9, France e-mail: \\ Xavier.Leoncini@cpt. univ-mrs.fr \\ 2 PIIM, Université de Provence-CNRS, Centre Universitaire de Saint Jérôme, F-13397 Marseilles, France e-mail: \\ Olivier.Agullo@up.univ-mrs.fr
}

the date of receipt and acceptance should be inserted later

\begin{abstract}
A numerical method is proposed in order to track field lines of three-dimensional divergence free fields. Field lines are computed by a locally valid Hamiltonian mapping, which is computed using a symplectic scheme. The method is theoretically valid everywhere but at points where the field is null or infinite. For any three dimensional flux conservative field for which problematic points are sufficiently sparse, a systematic procedure is proposed and implemented. Construction of field lines is achieved by means of tracers and the introduction of various Hamiltonians adapted to the "geometrical state" each line or tracer is. The states are artificially defined by an a priori given frame of reference and Cartesian coordinates, and refer to a Hamiltonian which is locally valid at the time step to be computed. This procedure ensures the preservation of the volume (flux condition) during the iteration. This method is first tested with an ABC-type flow. Its benefits when compared to typical Runge-Kutta scheme are demonstrated. Potential use of the method to exhibit "coherent" Lagrangian structures in a chaotic setting is shown. An illustration to the computation of magnetic field lines resulting from a three-dimensional MHD simulation is also provided.
\end{abstract}

PACS. 0 5.45.Ac, 05.45.Pq, 47.52. + j, 47.65.Md

\section{Introduction}

With the constant increase of computing power, one is now able to simulate more and more precisely complex systems, among which three dimensional fluid flows. In order to tackle these simulations one is often bound to use an Eulerian perspective on flows or fields. However, if one is interested in transport properties, a Lagrangian perspective is often best suited [1. For instance the phenomenon of chaotic advection translates the fact that fluid trajectories are chaotic despite the laminar structure of the flow 22. In two dimensional flows chaotic advection has been studied extensively, as it offers the possibility to drastically increase the mixing properties of a given timedependent flow. If the flow is stationnary trajectories of passive tracers resume to field lines, and no chaos or mixing occurs in two dimensions. However when considering three-dimensional fields, field lines are generically chaotic for a stationary flow. Understanding the field line chaotic structures in these stationary flows can be therefore considered as a first step towards understanding transport

\footnotetext{
a Unité Mixte de Recherche (UMR 6207) du CNRS, et des universités Aix-Marseille I, Aix-Marseille II et du Sud ToulonVar. Laboratoire affilié à la FRUMAM (FR 2291).Laboratoire de Recherche Conventionné du CEA (DSM-06-35).
}

in these flows. Note also that anyhow, a passive tracer trajectory is locally tangent to the field line for the considered time, hence computing field lines correctly is as well a first numerical step in order to compute trajectories. This chaos of field lines has been investigated for a long time in plasma physics, especially when concerned with the conception of magnetically confining devices such as tokamaks 3. Indeed, the conception of such devices originates from the fact that charged particles are locally trapped by and along magnetic field lines. The chaos of field lines is therefore a crucial ingredient for the understanding and characterisation of plasma transport properties. Note although that in plasma devices, such that a tokamak, numerical studies of three dimensional magnetic field lines is simplified thanks to the toroidal geometry and a strong anisotropic magnetic field.

In this paper we propose a volume (flux) preserving numerical approach to the computation of field lines of three dimensional divergence free fields, while making no assumption on the anisotropy and geometry of the field. A first attempt in this direction has been proposed in [4], however such schemes are first order ones and are not generic to flux conservative fields. Field lines of conservative flux fields as we already mentionned, known to be generically chaotic in three dimensions (see for instance 
Xavier Leoncini et al.: From chaos of lines to Lagrangian structures in flux conservative fields

[5] for some nice illustrations and references). This property is often illustrated by means of a special local transformation: the equation of field lines can be written in a Hamiltonian form. These Hamiltonians belong to the class of $1-\frac{1}{2}$ degrees of freedom systems, which are known to exhibit generically chaotic behaviour. More specifically the field lines exhibit Hamiltonian chaos, implying some volume preserving constraints as well as "time-reversibility". These features make Hamiltonian chaos quite peculiar, no source nor sinks are possibles, neither are attractors, as such the dynamics does not have special asymptotics displaying for instance a fractal attractor.

Our strategy to compute field lines numerically while keeping these specific chaotic features is to use this Hamiltonian mapping and couple it to a symplectic scheme. The symplectic scheme ensures the preservation of the flux of the original field, by preserving the volume in phase space 6. We insist, that this volume preservation is essential in Hamiltonian dynamics as it prevents the system from having any attractor as well as source or sinks in phase space, a feature which is essential when considering transport properties for large times and computing the kinetics limit. In the same spirit, we may expect that paying attention to volume preservation while computing field lines becomes quite relevant when the field itself is "properly" computed. We might think therefore to couple this numeric scheme for field lines to an exactly conservative integrator for the Euler flow, such as the one discussed in 7. Finally we would like to mention that the full three dimensional incompressible and ideal hydrodynamics can be also reduced to a Hamiltonian formalism [8, using for instance Clebsch variables, but we are then dealing with an infinite dimensional phase space, the numerical algorithms are then not easily implemented although using the so called "vortex-line representation" seems promising [9]. Our approach is much more modest as we do not deal with the computation of the field itself, but also allows us to consider any divergence free field, such as a magnetic field for instance.

Another potential interest in computing field lines for a flux free field is the possibility to compute Poincaré sections of the field lines. We are then able to represent global information regarding the three dimensional field on a plane or a number of planes, offering de facto another perspective on the flow and a tool from which physical phenomena may have a simpler or different explanation. In fact, this point of view has been recently used quite successfully in the context of identification of threedimensional reconnective structures of a plasma in a peculiar geometry [10, which were ill-defined in a Eulerian context. With such an approach, we should also be able to discriminate the physical importance of islands of regular motion within a stochastic sea in the Poincaré sections: For instance, by identifying the border between chaotic and non-chaotic zones, we might identify a localised three dimensional structure which does not necessarily have an Eulerian counterpart. If the Eulerian counterpart exists, we might have a tool to define clearly its shape and we might test, as another step, its dynamic robustness.
The paper is organised as follows. In Section 2 a brief summary of field line equations and the Hamiltonian mapping is given. In Section 3. we present how the Hamiltonian formalism is applied to numerical data. Then in Section 4 we apply the method to two different examples: First an ABC like flow is considered. The field lines are computed with a symplectic scheme and the Hamiltonian formalism and are compared with a Runge-Kutta integration, short-comings of the latter are clearly exhibited. A few different choices of parameters are then chosen for the ABC flow, and some three dimensional structures are extracted. Finally as a proof of feasibility beyond toy models, the method is applied to a magnetic field computed directly from an MHD simulation and Poincaré sections are shown.

\section{Basic Equations}

\subsection{Equation of field lines}

Let us briefly recall the definition of field lines, for this purpose let $\mathbf{v}$ be a three-dimensional vector field. Field lines of $\mathbf{v}$ are curves which are tangent to the field at any point. This definition may be problematic when the field is zero valued at one point, however we may expect for a reasonable smooth field that these points if they exist may live on a subset of zero measure (their union have a zero volume). In the following we will consider that the computed field lines do not cross such a point. In a mathematical sense we may consider the definitions of field lines as:

$$
\mathbf{v} \wedge d \mathbf{M}=\mathbf{0}
$$

where $d \mathbf{M}$ stands for a small displacement along the field line around a point $M$. It is usually easier to consider a given reference frame and use coordinates, we then rewrite Eq.(1) as

$$
\frac{d x}{v_{x}}=\frac{d y}{v_{y}}=\frac{d z}{v_{z}}=\frac{d s}{v}
$$

where $d x, d y, d z$ are the coordinates of the displacement in a given frame, and $v_{x}, v_{y}, v_{z}$ are the coordinates of $\mathbf{v}(M)$ and $v$ is its norm; $d s$ stands for the norm of $d \mathbf{M}$ (we assumed an orientation of the line is given ). Let us consider now a smooth field, we then choose our coordinate systems such that in a given region $v_{z} \neq 0$. Equations (2) are directly reduced into a system of two ordinary differential equations

$$
\left\{\begin{array}{l}
d x / d z=v_{x} / v_{z} \\
d y / d z=v_{y} / v_{z}
\end{array},\right.
$$

where $z$ acts as a time variable. Note that this reduction is only locally valid as it may induce some fictitious singularities if the line ends up in the neighbourhood of points where $v_{z}=0$. This point will be discussed again later (see sec 3.3. Let us now focus on the Hamiltonian formalism. 


\subsection{Hamiltonian formalism for divergence free fields}

We are interested in finding a transformation which will allow one to describe the evolution of tracers along a field line in a Hamiltonian formalism. So let $\mathbf{v}$ be a three dimensional divergence free field, such as for instance the velocity field of an incompressible flow or a magnetic field and let us reformulate Eq.(3) into a Hamiltonian formalism in this frame. The divergence free condition

$$
\nabla \cdot \mathbf{v}=0
$$

implies the existence of a potential vector $\xi$ such that

$$
\nabla \wedge \xi=\mathbf{v}
$$

$\xi$ being defined up to a given arbitrary gradient (gauge condition).

Let us consider the vector potential $\xi$ and relabel its coordinates $\xi_{x}=-p, \xi_{z}=H(x, y, z)$. Now using the gauge liberty on the vector potential $\xi$, we set $\xi_{y}=0$. Given Eq.(15) the field $\mathbf{v}$ is rewritten as follows

$$
\mathbf{v}=\left(\frac{\partial H}{\partial y},-\frac{\partial H}{\partial x}-\frac{\partial p}{\partial z}, \frac{\partial p}{\partial y}\right) .
$$

Notice taht the equation of motion for the Hamiltonian $H(x, y, z)$ are non-canonical. In what follows, we change the coordinate system such that they become canonical. Given the coordinates $(x, y, z)$, we define $q=x$ and $\tau=z$ and switch to the new system $(q, p, \tau)$. We then consider the function $\widetilde{H}(p, q, \tau)=H(x, y, z)$. Before moving on, we recall that the change of coordinates implies

$$
\left\{\begin{array}{l}
\partial_{x} f=\partial_{q} \tilde{f}+\partial_{x} p \partial_{p} \tilde{f} \\
\partial_{y} f=\quad v_{z} \partial_{p} \tilde{f}
\end{array}\right.
$$

where $\partial_{a} f=\partial f / \partial a$. We then rewrite the equations of the field (3) in the new coordinate system and readily obtain from Eqs. (6)

$$
\left\{\begin{array}{l}
\dot{q}=\frac{\partial H}{\partial p} \\
\dot{p}=-\frac{\partial H}{\partial q}
\end{array}\right.
$$

where the dot refers to a derivative with respect to $\tau$ and the tilde was removed from $H$. The equations are of canonical Hamiltonian form with the $(p, q)$ acting as canonical conjugates, and $\tau$ acting as a time variable. Hamiltonian $H$ is the component of the potential vector associated with the chosen fictitious time (the $z$-direction in our case).

\subsection{Locality}

Note that the singularity for $v_{z}=0$ is not apparent in Eq.(8). However the singularity remains because the fictitious time becomes singular for $v_{z}=0$, which if not cared about might reverse the arrow of "time". This transformation is actually only locally valid. However, first, given our hypothesis of a relatively smooth field, it is unlikely that we hit a $\mathbf{v}=\mathbf{0}$ point. Second, we have a free choice of our coordinate system, thus we can rotate our frame as we think is best. With this freedom of transformation, a natural choice would be to consider a locally well suited coordinate system in order to avoid singularities in $v_{z}$. Unfortunately, this strategy is not well suited when computing many field lines numerically at the same time. Indeed each would need its own local Hamiltonian since the gauge condition will be point dependent. And, as a result this would be numerically expensive.

Besides the singularity, injectivity of the transformation $(x, y, z) \rightarrow(q, p, \tau)$ must be ensured if one wants to perform time evolution in Hamiltonian variables. Indeed, the momentum is formally defined by

$$
p=\int^{y} v_{z} d y,
$$

and, without more specifications, unicity in the determination of $y$ given the triplet $(q, p, \tau)$ is not guaranteed: the history of $v_{z}$ along the field line is included in $p$ and we may recover problems dealt with previously, related to $v_{z}=0$. Moreover, it is also obvious in Eq. (9) that another difficulty will arise in a region for which $v_{y}=0$. In this region we expect to have $d y=0$ and thus a stationary $p$ in $y$, making the transformation likely non-invertible.

As will be discussed in Sec. 3 we settled for a compromise between implementability as well as numerical cost and debugging time. Let us now discuss one of the key reasons for this transformation to be performed.

\subsection{Volume preserving scheme}

We will now use this Hamiltonian structure in order to compute numerically the field lines of $\mathbf{v}$, because it will allow the use of a symplectic scheme which ensures the preservation of invariants, and which given the divergence free condition (4), implies the conservation of volume along field lines.

It is well known that intrinsically to any source-free systems, there is a volume form of the phase space $\Re^{n}$, say

$$
f=d x_{1} \wedge d x_{2} \ldots \wedge d x_{n}
$$

such that the dynamic evolution preserves this form. This constraints the geometric structure of the field lines. To explicit the structure numerically, it is crucial to use a scheme preserving this property as shown in section 3

For $n=2$, source free vector fields are Hamiltonian fields and symplectic algorithms are area-preserving maps. The situation is different for $n \geq 3$ : all the conventional methods, including the well-known Runge-Kutta and Euler methods, are non volume preserving (see 611]). By means of an "essentially Hamiltonians decomposition" of source free vector fields, a volume preserving difference scheme was proposed in Ref. [6]. The algorithm we used we will restrict ourselves to the case $n=3$ - does not use the decomposition of Ref. [6]: in such a way, computation of the "time evolution" of the tracers along the field lines requires the explicit introduction of only one Hamiltonian (and not two), the price being the introduction of a time 
dependent preferential space direction (see Sec. 2.3). However, the idea is still to use the underlying local Hamiltonian structures, as explained in Sec.2.2 and integrate the dynamical equations (8), by means of symplectic schemes. This procedure, indeed, allows the preservation of the volume form

$$
d x \wedge d y \wedge d z=d q \wedge d p \wedge \frac{d \tau}{v_{z}} .
$$

The meaning of Eq. (11) is the following one : We know that $d x \wedge d y \wedge d z$ is preserved as time $t$ evolves. From the Hamiltonian framework we have that $d q \wedge d p \wedge d \tau$ is preserved as the fictitious time $\tau$ evolves which is here the coordinate $z$. The consistency is provided by the way we follow the evolutions, i.e., by the nonlinear relation $d z / d t=v_{z}$ between these two evolutions.

\section{Application to numerical data}

\subsection{Fourier representation}

We now present a possible way to implement this formalism when dealing with a field obtained by numerical simulation. We consider a quite common case of a field with periodic boundary conditions. If this is the case, it is a common practice to consider the Fourier space for the computation as for instance when one uses a pseudospectral method. We therefore consider a case where all the Fourier components $\hat{\mathbf{v}}_{\mathbf{k}}$ of $\mathbf{v}$ are known. Here the vector $\mathbf{k}$ is used as a mode index. We shall also for convenience consider that the number of modes is finite, which is more realistic when dealing with numerical data. Using equations (6) we then write the Hamiltonian and momentum in the $(x, y, z)$ coordinates:

$$
\begin{aligned}
H(x, y, z)= & \sum_{k_{y} \neq 0} \frac{\hat{v}_{\mathbf{k}, x} e^{i \mathbf{k} \cdot \mathbf{x}}}{i k_{y}}+y\left(\sum_{k_{y}=0} \hat{v}_{\mathbf{k}, x} e^{i \mathbf{k} \cdot \mathbf{x}}\right) \\
& -\sum_{k_{y}=0, k_{x} \neq 0} \frac{\hat{v}_{\mathbf{k}, y} e^{i \mathbf{k} \cdot \mathbf{x}}}{i k_{x}}-x\left(\sum_{k_{y}=0, k_{x}=0} \hat{v}_{\mathbf{k}, y} e^{i \mathbf{k} \cdot(1)}\right) \\
p= & \sum_{k_{y} \neq 0} \frac{\hat{v}_{\mathbf{k}, z} e^{i \mathbf{k} \cdot \mathbf{x}}}{i k_{y}}+y\left(\sum_{k_{y}=0} \hat{v}_{\mathbf{k}, z} e^{i \mathbf{k} \cdot \mathbf{x}}\right)
\end{aligned}
$$

Note that the condition $\xi_{y}=0$ is not fully sufficient to determine $H$ and $p$, and the equations (13) and (14) which are defined up to a function of $x$ and $z$, reflect our choice of trying to keep $p$ as simple as possible, leaving the "complexity" in $H$.

\subsection{Numerical scheme}

In order to compute field lines, we take advantage of the Hamiltonian formalism and use a symplectic scheme. In the present case we consider the fourth and sixth order Gauss-Legendre scheme proposed in [12]. However the trans- tion

formation from the $(x, y, z)$ to $(p, q, \tau)$ is not trivial. For instance, having access to the function $\widetilde{H}(p, q, \tau)$ in order to compute the Hamiltonian dynamics seems rather complex. In order to avoid these difficulties, even though the field lines are propagated in the $(p, q, \tau)$ space, we decided to compute the $-\partial_{q} \tilde{H}$ in the $(x, y, z)$ space using the expressions (7).

We therefore have to keep a constant correspondence between the two spaces. This is performed by considering the expression of $p$ given by Eq.14), and explains our gauge choice to keep it as simple as possible. In order to get $(x, y, z)$ given a value of $(p, q, \tau)$, only $y$ is given implicitly by Eq.(14) since $x=q$ and $z=\tau$. The inversion is done numerically using a simple Newton scheme, for which the first guess for the root is given by the Euler method.

\subsection{Dealing with pseudo-singularities}

Up to now we have assumed that the Hamiltonian transformation is globally valid. By the choice of coordinate system, the direction $z$ acts as a time variable, and is therefore assumed to be a monotonic function along the field line. One may therefore wonder what happens when we hit a fold, which turns the field line in the reversed direction. If this is the case $z$ can not be monotonic and the Hamiltonian transformation fails. Our way of dealing with such problems is to assign a state to each field line, corresponding to which direction the line is propagated in. Indeed when we reach a point where $v_{z}=0$, one may expect that $v_{x}$ or $v_{y}$ is not null. Since our coordinate system is arbitrary, we may conceptually rotate our frame in order to get $v_{z} \neq 0$ in this new coordinate system and propagate our field with the symplectic scheme. Ideally, it would be best suited to locally rotate the coordinate system and try to take the arc-length along the line as a measure of the fictitious time variable. However when (12)computing many field lines together, this procedure would mean to keep track of all coordinate systems and rotate them after each step to the local Frenet system before performing the transformation and the next time step. This seems numerically expensive. We settled for a more pragmatic point of view. Field lines are thus assigned a number $i \in\{1,2,3\}$ corresponding to which coordinate system the (14) Hamiltonian transformation is applied to. These numbers corresponding to a cyclic permutation of the coordinates $(x, y, z)$ of a coordinate system given once and for all.

Furthermore as was previously discussed, problems may also arise when $v_{y}=0$. If such a seldom situation occurs, we simply permute the current $x$ and $y$ directions. Formally this corresponds to a different choice for $p$ and $q$ and a different choice of gauge for $\xi$. A new number $j \in\{1,2\}$ is therefore assigned to the field line.

When changing the coordinate system, we have to make sure that we will continue to "advance" along the line, meaning we have to be careful and make sure that the new Hamiltonian transformation will not end up in going backtrack along the already computed path. We therefore choose an orientation for the lines and impose the condi-

$$
\mathbf{v} \cdot d \mathbf{M}>0
$$


It is obviously important to ensure that this condition is still satisfied when changing coordinates. Since it is conceptually easier to deal with a positive time step $d \tau$, and since field lines are invariant when multiplied by a scalar field, we perform the change $\mathbf{v} \rightarrow-\mathbf{v}$ when necessary in order to comply with Eq.(15). This implies that another number $k \in\{1,2\}$ must be assigned to the field line.

At last, since we try to avoid performing a rotation of our coordinate system, except for simple permutation of the coordinates, we may hit points for which the field is along one of the coordinates, leaving us with two zerovalued coordinates and problems of invertibility. In order to deal with such situations, we actually have to consider a second reference frame, not issued by a permutation. We may then consider for instance a $\pi / 4$ rotation around the $z$-direction of the original coordinate system, and deal with problematic points in this frame. Given all possibilities while computing the field line, its state (the numbers $(i, j, k))$ can thus take twelve different values, and we can be in two different coordinate systems, giving us a broad range of twenty four Hamiltonians in order to compute a field line.

\section{Applications}

In order to test the algorithm and start to explore the possibilities offered by the analysis of field line chaos, we started with a basic three dimensional flow, namely the ABC-flow 1314. We first validate our numerical scheme by considering a choice of parameters for which no singularity occurs and the Hamiltonian transformation is global. Further on, we show the advantages of using a symplectic scheme over a non-symplectic one. We then consider integrable and chaotic flows for which singularities occur. In the latter case, Lagrangian structures are exhibited as a result of the analysis of Poincaré sections. Finally as a proof of concept we compute the magnetic field lines, in the context of the dynamo process, of a magneto-hydrodynamic flow.

\subsection{ABC flow}

In order to test the algorithm we consider the following periodical flow which may be seen as a particular example of the well know ABC flow 1314]:

$$
\begin{aligned}
& v_{x}=\cos y-\varepsilon \sin z \\
& v_{y}=\sin x+\varepsilon \cos z \\
& v_{z}=\cos x-\sin y+v_{0 z},
\end{aligned}
$$

where $\varepsilon$ and $v_{0 z}$ are parameters.

We started to benchmark the code by comparing its results with the one obtained using a non symplectic fourth order Runge-Kutta scheme on Equations (3). Note that in order to have some consistency, comparison is done with the symplectic fourth-order Gauss-Legendre integrator [12]. For this first test we took $\varepsilon=0.9$ and $v_{0 z}=4$ in order to avoid singularities which occurs at points where $v_{z}=0$. Since $v_{z}>0, z$ is used as the time variable, and due to the periodic character of the flow, we can reduce our analysis of the flow on the Poincaré map defined by the crossing of field lines with the successive $z=0[2 \pi]$ planes. The resulting plot is depicted in Fig. 1using the two methods. The "time step" used for the run was $\delta z=\pi / 100$. To the naked eye, the same section is obtained for both cases, points solely differ in the chaotic region. The chaotic behaviour of steady field lines in three dimensions is clear. This first test is used in order to confirm that the proposed method computes what it is expected to.

Now let us consider the advantages of using such a symplectic algorithm. For this purpose we consider the integrable case $\varepsilon=0$, and we compare the evolution of a field line starting from the same initial condition (for visual purposes) but computed using both methods. Since the flow is integrable we expect to see a closed line on the Poincaré map.

First we consider both cases with an identical relatively large time step $\delta z=\pi / 10$. The results are shown in Fig. 2 and clearly demonstrate that the non symplectic method has some shortcomings as the expected closed line becomes a cloud of points, and inward diffusion is observed. Now in order to be "fair", since the non symplectic scheme is almost twice faster on an identical computer than the "conserving" one, we took a smaller time step $\delta z=\pi / 20$ for the Runge-Kutta method in order to match the integration times (CPU times) between the methods. Still, as seen in the insert in Fig. 2 some inward diffusion is observed with the Runge-Kutta scheme. These non-conservative features may induce spurious effects, for instance when considering transport properties in the kinetic limit, while transport may be not Gaussian (see for instance 1617) one may end observing a diffusive behaviour with a diffusion coefficient governed by the algorithm, as this diffusive behaviour will end up erasing in a finite time any potential memory effect generated by the presence of regular trajectories in a mixed phase space. In this sense using a conservative algorithm despite its extra numerical overhead, we may avoid false interpretations which may arise with Runge-Kutta as for instance possible transient anomalous behaviour.

In the rest of the paper we use the sixth-order GaussLegendre scheme.

\subsection{Flow with pseudo singularities}

We now test the code on a flow for which the problems of singularities and non invertibility occur. Namely we consider a series of flows for which $v_{0 z} \rightarrow 0$. We first consider the integrable case $(\varepsilon=0)$. The results are shown in Fig. 3. It is easy to see from Eqs.(16) that the flow becomes somewhat two-dimensional, as the $z$ dependence of the flow completely vanishes. For this "two-dimensional" steady flow $\cos x+\sin y=H_{2 D}$ is a constant along the field line and corresponds to the stream function which acts as a Hamiltonian. This property gives rise to the closed lines observed in the Poincaré section depicted in Fig. 3. Thus 
for any value $v_{0 z}>0$ we expect to have identical sections, which is the case as we take different values of $v_{0 z}$ ranging from 3 to $0.01\left(v_{0 z} \rightarrow 0\right)$ observed in Fig. 3. An additional feature occurs when $v_{0 z}=0$, all field lines appear to become periodic and localised (forming a closed line). In this regard, we like to pinpoint a specific fact about our choice for the equations of the $\mathrm{ABC}$ flow given by Eqs. (16). In fact it is usually a common procedure to take $v_{z}=H_{2 D}(x, y)$ (see for instance [1518]). In the integrable situation this procedure leads to a constant $v_{z}$, and helical field lines. In these regards, the choice of the minus sign in the third equation of Eqs. (16) drastically changes the behaviour of field lines. From the perspective of testing the numerical scheme, this choice also proved to be more challenging than usually [15], since the field lines "bite their tails".

Since the numerics are giving us what we expect, we may now move to a non-integrable situation. For this purpose we consider $\varepsilon=0.15$ with a $v_{0 z}=0$ : the integrable case with closed field lines is perturbed. A Poincare section is shown in Fig. 4. One can observe regions with regular quasi-periodic field lines, and regions of chaos. In the regular region, one can notice some delocalised field lines, as well as apparently localised ones (at least in the $z$ direction). In the chaotic sea, one notices a region with a vanishing density of points, in other words a region which is not often crossed. Since the depicted section is computed in the $x=0[2 \pi]$ plane, one can expect that regions in these planes for which $v_{x}=0$ are not crossed. It implies that regions around the curves given by $\cos y=\varepsilon \sin z$ are not often crossed. These curves correspond to the light region in Fig. 4 for one, and for the "centre" of the regular region in Fig. 4 for the other one. Before concluding on this particular example, we want to mention the existence of two islands in the chaotic region nearby the low-density region that the method is able to detect.

\subsection{Lagrangian structures}

In fact the Poincaré section shown in Fig. [4 is quite generic for systems with one and a half degrees of freedom, with regions of regular motion and a chaotic sea. In this section we describe the shape of the field lines corresponding to the regular regions. Indeed contrarily to a true phase space section from a given Hamiltonian, the section depicted in Fig. 4 corresponds to field lines obtained typically by a "product" of local Hamiltonians. The "time" dependence is therefore not always monotonic. We thus may expect more complexity than the typical braid of helical structures which is expected in the phase-space extended with a time direction. Besides this possible increase of complexity, the regular structures may have also a crucial physical impact. For instance, if one consider the field $\mathbf{v}$ as being the velocity field of an incompressible fluid, the transport properties of passive scalars will be along the field lines. Thus the properties of these regular regions may greatly influence global properties of transport or mixing in this flow.
In order to localise the coherent Lagrangian structures associated with regular field lines, we just consider initial conditions within a regular zone in the Poincaré section, and computed the resulting field lines. To our knowledge this type of methodology has not been applied to generic divergence free fields, in particular to the considered $\mathrm{ABC}$ flow. Results are shown in Fig. 5. Typically one notices two types of behaviours, localised field lines, which are drawing a finite surface, and unlocalised ones. Note the mushroom-like shape of the unlocalised field line, which if considered as the trajectory of a passive tracer, may appear as successive sheets in an experiment. Among the unlocalised regular field lines, one can also find a simple helical like form, when one considers an initial condition within the two small islands located not far from the no crossing region. To conclude on these structures, it is worthwhile mentioning that even by looking at the sections trough different planes $(y=0[2 \pi]$ or $z=0[2 \pi])$ the same structures are obtained. They just have different sections with these planes. One may also infer the Poincaré plot depicted in Fig. 目 that the localised structures (Fig. 5 lower panel) appear as sandwiched between the unlocalised mushroom ones (Fig. [5 upper panel). We may also note that the observed "coherent structures" do not have necessarily a simple tubular shape, hence we may speculate that using this technique to uncover spatially extended coherent structures in more complex flows such as MHD, may yields some unexpected shapes with maybe important physical consequences and selection mechanisms.

\subsection{Magnetic Field from MHD}

We shall now consider a full feature numerical study and move on to the magnetic field obtained through magnetohydrodynamics (MHD) simulations. The origin of cosmic magnetic fields can be investigated in the frame of MHD. The problem is to extract the underlying mechanisms of the dynamo process, i.e. of the self-excitation of a magnetic field by plasma or fluid motions. To describe the generation of large-scale magnetic fields from small-scale turbulence many mechanisms are involved and, in the following, we will focus on the so-called $\alpha^{2}$-dynamo (see for instance [20]).

The equations of incompressible MHD, written in the usual units, read

$$
\begin{aligned}
\frac{D}{D t} \mathbf{u} & =-\nabla \mathbf{p}+\mathbf{b} \cdot \nabla \mathbf{b}+\nu \nabla^{\mathbf{2}} \mathbf{u}+\mathbf{f} \\
\frac{D}{D t} \mathbf{b} & =\mathbf{b} \cdot \nabla \mathbf{u}+\eta \nabla^{\mathbf{2}} \mathbf{b} \\
\nabla \cdot \mathbf{b} & =\nabla \cdot \mathbf{u}=0
\end{aligned}
$$

where by definition, $\eta$ is the magnetic diffusivity, $\nu$ the kinematic viscosity and the scalar $p$ is the sum of the hydrostatic and the magnetic pressures. We choose $\nu=\eta=$ $10^{-3}$. Here, both fluid velocity $\mathbf{u}$ and magnetic field $\mathbf{b}$ are expressed in Alfvèn speed units. The flow is forced by a divergence free field at scales much smaller than the width $L=2 \pi$ of the cubic and periodic box. $\alpha^{2}$-dynamo is enhanced by imposing the forcing to be maximally helical: 
its relative helicity is one at any time 1920 . Initial conditions are $\mathbf{u}=0$ and $\mathbf{b} \sim \mathbf{0}$. The flow is driven by the forcing and it turns out that the Reynolds number based on the Taylor microscale is around a few units: this is a moderate turbulent regime. In fact, a noteworthy effect of the Lorentz force is to decrease by roughly a factor ten the Reynolds value. In Fig. 7 are drawn time evolutions of the total kinetic and magnetic energies of the plasma. Clearly, owing to the kinetic forcing, kinetic energy is initially rapidly accumulated. The growth of the magnetic energy initiates then a decrease of the kinetic energy and a quasi-saturated state is reached at time $T=300$. The eddy turnover time is typically of a few time units. Transfer of helicity from the forcing to the flow and from the flow to the magnetic field, but also Alfvén effects and an inverse cascade process (after time $T \sim 15$ here) lead to the generation of a robust anisotropic large scale magnetic field, characterised by a pile up of magnetic energy at the largest scales [2120]. Once the large scale magnetic field reaches a stationary state, a rough snapshot of the Eulerian field can be summarised by the approximation

$$
\mathbf{b} \sim \cos z \mathbf{e}_{\mathbf{x}}+\sin z \mathbf{e}_{\mathbf{y}}+\epsilon \sum_{|\mathbf{k}| \sim k_{F}} \mathbf{b}_{\mathbf{k}} \exp (i \mathbf{k} \cdot \mathbf{x})
$$

where $\epsilon$ is a small parameter to indicate that energetically dominant modes are at the largest scales $k=2 \pi / L=1$ and $k_{F}$ is the forcing scale. In Fig. 7 we draw a Poincaré section of the three-dimensional magnetic field lines resulting from the magnetic field obtained at time $T=900$. This is the section of 100 magnetic field lines. Initial positions of the tracers to determine the fields lines are randomly distributed in the periodic box. The computation is done by including in the field an energetically consequent fraction of the modes. Inclusion of all the modes would have been too computer time consuming and, since in this paper we put our effort on showing a proof of concept and we do not focus on the details of the Lagrangian structures. For instance, dissipative Eulerian structures are not included in the structure of the field. The existence of chaotic Lagrangian structures is however clearly observed in Fig 7. It is also observed a"drift of the structures" according to their height $z$. In fact, this drift trivially originates from the Eulerian field as can be seen by inspection of the first term on the r.h.s of Eq. (18). We like to insist on the fact that Fig. 7 clearly suggests the existence of a non trivial underlying Lagrangian and chaotic organisation in duality with the well established coherent Eulerian structures suggested by the approximation (18). These remarks suggest that the study of the Lagrangian structures in this type of flow offers promising prospects. Indeed, chaos of field lines of a flow, neither necessarily turbulent nor unstationnary, is an ingredient known to clearly enhance a dynamo effect; an clear example being the G.O. Roberts dynamo where an ABC flow is used 22. The large scale term of the saturated magnetic field in the simulations is, in fact, also of ABC type. However the role of the turbulence in the generation of the former, but also how the inverse cascade generates helical structures in a turbulent flow are not clear. Thinking those processes in terms of Lagrangian structures in parallel with a classification and measure of their helicities might allow a new understanding of the interplay of turbulent, helical and chaotic phenomena in dynamo processes. Finally, we should emphasise that the presence of chaos does not necessarily rely onto any complexity in the small scale perturbative term of Eq. (18). Indeed, it probably essentially results in the presence of the large scale Beltrami structure, $\mathbf{b}_{\epsilon=0}=\kappa \nabla \wedge \mathbf{b}_{\epsilon=0}$ with $\kappa=-1=$ Cte, as a consequence of the Arnold theorem [13]. We may also emphasise on the fact that the existence of chaotic field lines is in turn a signature of the three dimensional nature of the field, and considering the anti-dynamo theorems in two-dimensional flows we may expect that the mechanisms underlying the dynamo effects are generically correlated with the existence of chaotic field lines

\section{Conclusion}

The main purpose of this work is to propose a way to compute field lines of divergence free fields, while keeping the conservative flux condition valid. Field lines are computed by performing a locally valid Hamiltonian transformation, which in turn is numerically computed using a symplectic scheme. The method is theoretically valid anywhere but at points where the field is null or infinite. For fields for which such points are sufficiently sparse that we consider they numerically do not exist, a systematic procedure has been proposed. Field lines are propagated using up to twenty four different Hamiltonians, depending in which "state" each line is. The states being artificially defined by an a priori given frame of reference and Cartesian coordinates, and refers to which Hamiltonian is currently locally valid for the time step to be computed. The potential application to three dimensional fields with periodic boundary conditions is discussed and explicit usable expressions for the Hamiltonian and momentum are given. We have also provided a detailed possible actual potential implementation into a numerical code of the ideas, and presented numerical results obtained for specific systems. In this procedure, we have shown some advantages of using this Hamiltonian-symplectic formalism over a regular Runge-Kutta scheme for the field lines.

Moreover, while testing the numerical scheme, field lines of $\mathrm{ABC}$ type flows have been investigated. For a considered integrable flow with no mean flow, we have shown that field lines can be all closed. Also a Poincaré section of a non-integrable flow, for which the field lines can not be described by a global Hamiltonian has been computed and a mixed phase space with a chaotic sea and regular regions is exhibited. From this analysis Lagrangian coherent structures are extracted, and either localised or extended structures have been found. Finally in order to show an application of the numerical tool in order to tackle physical problems, the field lines of the magnetic field resulting from an magneto-hydrodynamic simulations in a dynamo process have been computed. 
We would like to thank G. M. Zaslavsky for fruitful discussions during the overall process of this work, we also acknowledge useful comments from the Non linear Dynamics Group at CPT.

\section{References}

1. A. A. Chernikov et al., Phys. Lett. A 144, 127 (1990).

2. H. Aref, J. Fluid Mech. 143, 1 (1984).

3. R. White, The Theory of Toroidally Confined Plasmas (World Scientific - Imperial College Press, 2001).

4. M. Z. Qin, and W. J. Zhu, Comp. Math. Applic. 26, 32-42 (1993).

5. V. N. Govorukin, A. Morgulis, V. I. Yudovich, and G. M. Zaslavsky, Phys. Rev. E 60, 2788 (1999).

6. F. Kang and S. Zai-jiu, Numerische Mathematik 71, 451 (1995).

7. B. A. Shadwick, J. C. Bowman, and P. J. Morrison, SIAM J. Appl. Math. 59, 1112 (1999).

8. I. Antoniou and G. .P. Pronko, Theoretical and Mathematical Physics 141, 1670-1685 (2004).

9. E. A. Kuznetsov, J. Nonlinear Math. Phys. 13, 64-80 (2006).

10. D. Borgogno and D. Grasso and F. Porcelli and F. Califano and F. Pegoraro and D. Farina, Phys. Plasmas 12, 032309 (2005)

11. E. Hairer, C. Lubich, and G. Wanner, Geometric Numerical Integration (Springer-Verlag, Berlin, 2002).

12. R. McLachlan and P. Atela, Nonlinearity 5, 541 (1992).

13. V. I. Arnold, C. R. Hebd. Séances Acad. Sci. 261, 17 (1965).

14. S. Childress, J. Math. Phys. 11, 3063 (1970).

15. G. M. Zaslavsky, R. Z. Sagdeev, D. A. Usikov, and A. A. Chernikov, in Weak Chaos and Quasi-Regular Patterns, edited by B. Chirikov, P. Cvitanovic, F. Moss, and H. Swinney (Cambridge University Press, 1991).

16. X. Leoncini, O. Agullo, S. Benkadda and G. M. Zaslavsky, Phys. Rev. E 72, 026218 (2005).

17. X. Leoncini, L. Kuznetsov and G. M. Zaslavsky, Phys. Rev. E 63, 036224 (2001).

18. O. Agullo, A. Verga, and G. M. Zaslavsky, Phys. Rev. E 55, 5587 (1997).

19. O. Agullo and S. Benkadda, Commun. Nonlinear Science and Numerical Simulation 8, 455 (2003).

20. A. Brandenburg, Astrophys. J. 550, 824 (2001).

21. D. Biskamp, Nonlinear Magnetohydrodynamics (Cambridge University Press, Cambridge, 1993).

22. A.D. Gilbert, Dynamo Theory In: Handbook of Mathematical Fluid Dynamics, volume 2, pages 355-441 (Elsevier, 2003).

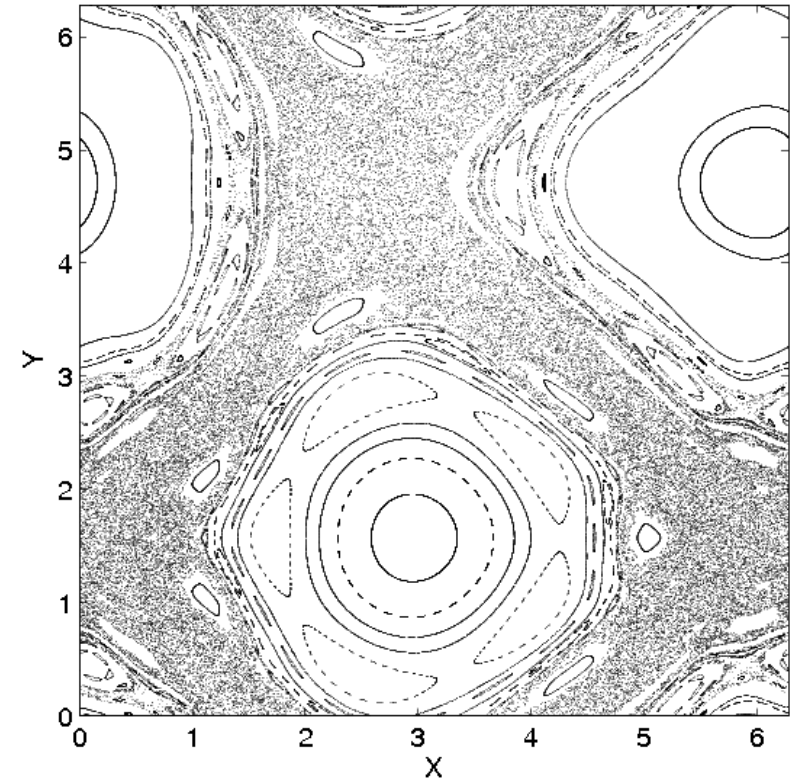

Fig. 1. Poincaré section of the flow (16) with $v_{0 z}=4$ and $\varepsilon=0.9$. The Poincare section is performed on the $z=0[2 \pi]$ plane. The flow is computed using the 4th-order symplectic scheme with a time step $\delta z=\pi / 100$.

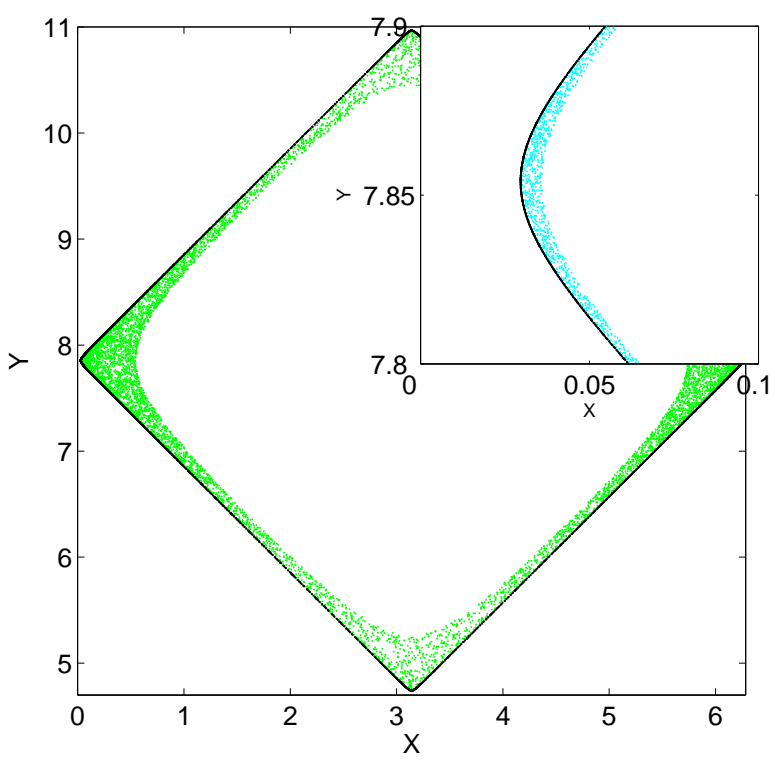

Fig. 2. Poincaré section of the flow with $v_{0 z}=3$ and $\varepsilon=0$. The Poincare section is performed on the $z=0[2 \pi]$ plane for only one initial condition $(3.142,4.742) \sim(\pi, 3 \pi / 2)$. The black line corresponds to the expected quasi-periodic trajectory obtained with the fourth-order symplectic scheme. The cloud of green dots corresponds to the trajectory obtained by the fourth-order Runge-Kutta scheme, showing some diffusion towards the centre of the island. Integration is performed up to $z=\pi 10^{6}$, with a time step $\delta z=\pi / 10$. In the insert the same simulation is performed for the Runge-Kutta scheme but with a smaller time step $\delta z=\pi / 20$ diffusion is still present, the dark line being a replot of the results obtained with the symplectic scheme and the larger time step $\delta z=\pi / 10$. 

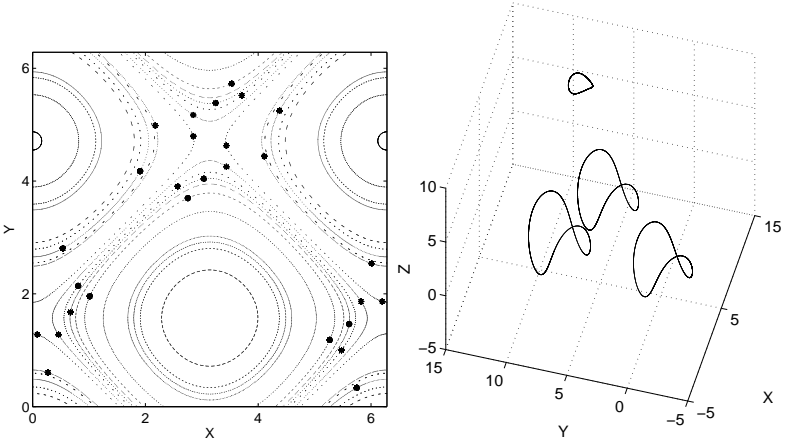

Fig. 3. Left: Poincaré section of the flow (16) for the integrable case $\varepsilon=0$ : note that the same section is obtained as we take different values of $v_{0 z}$ ranging from 3 to $0.01\left(v_{0 z} \rightarrow 0\right)$. The * signs, correspond to points obtained for $v_{0 z}=0$, the field lines appear to be periodic for this case. The Poincaré section is performed on the $z=0[2 \pi]$ plane with $\delta \tau=\pi / 50$. Right: Four field lines computed for the integrable case $\varepsilon=0$ and $v_{0 z}=0$. Field lines are indeed periodic and form closed lines.

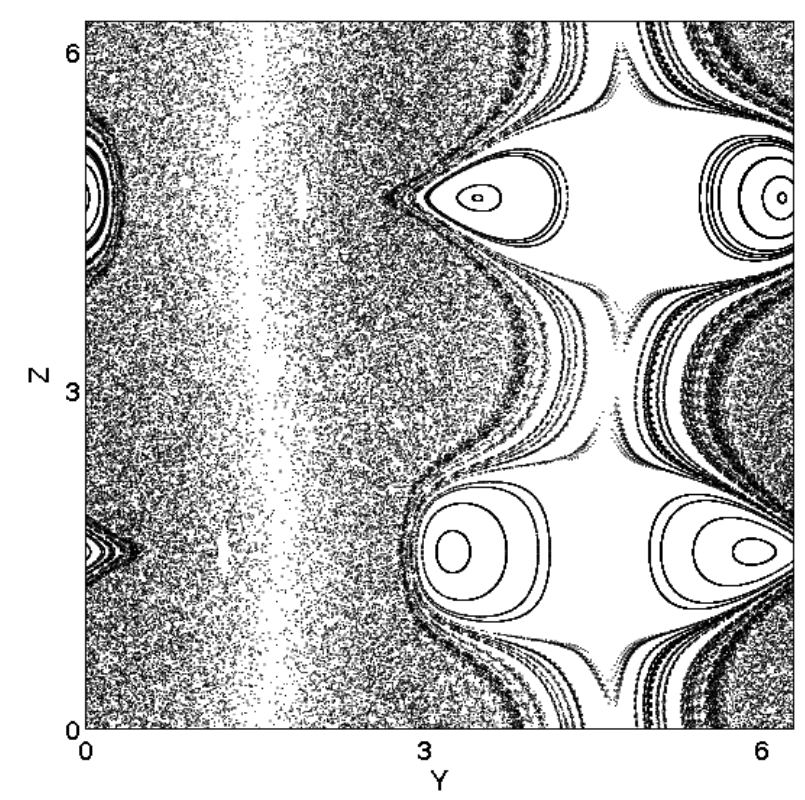

Fig. 4. Poincare section of the flow 16 for a chaotic case $\varepsilon=0.15$, for a flow with pseudo-singularities. One can infer a chaotic region and a regular one. One also notices a region which is not often crossed in the chaotic sea (lighter zone). The Poincaré section is performed on the $x=0[2 \pi]$ plane. The plot is made with 100 field lines which are iterated $210^{5}$ times with a time step of $\delta z=\pi / 100$.
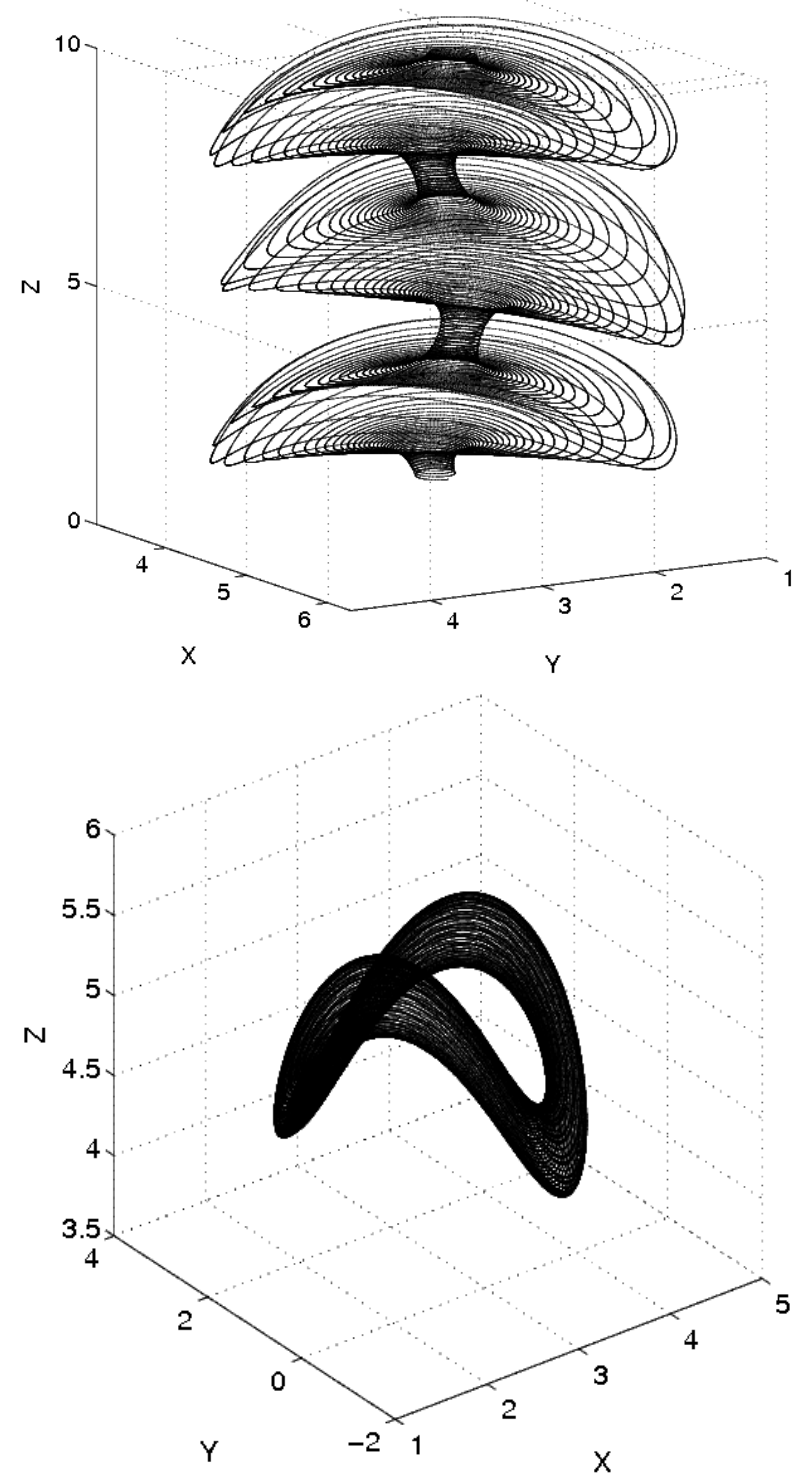

Fig. 5. Upper panel: regular drifting field line. Lower panel: Localised field line.

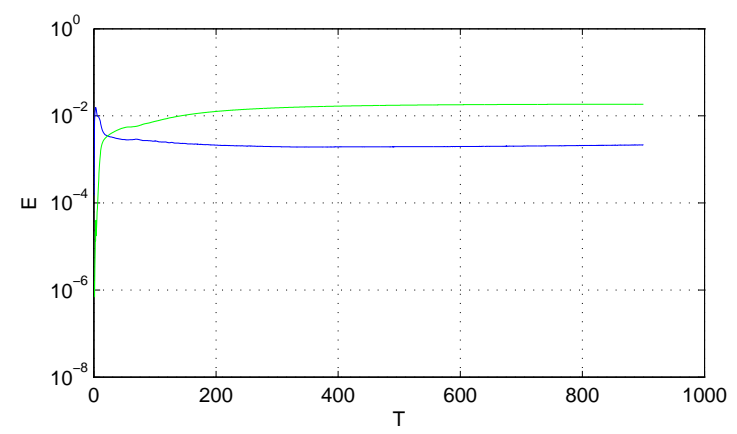

Fig. 6. Time evolution of kinetic energy (green line) and magnetic energy (blue line). 


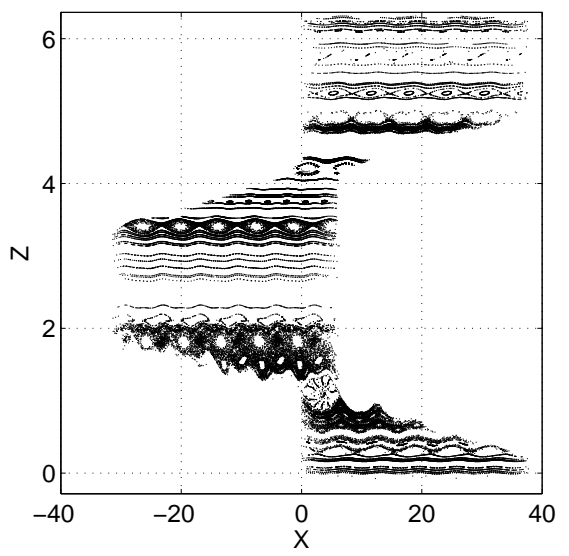

Fig. 7. Poincaré section of magnetic field lines. 